\title{
Estrategias de aprendizaje y rendimiento académico universitario: Una mirada desde los estudiantes de tecnológicos públicos ${ }^{1}$
}

\author{
Amaya Sauceda, Rosas Amadeo ${ }^{2}$ \\ Rafael Sánchez, Áurea Elizabeth ${ }^{3}$
}

\section{Resumen}

La educación superior en los tecnológicos públicos, propicia un espacio de interacción donde las ideas que se generan, son debatidas y profundizadas por los docentes, quienes al mismo tiempo hacen uso de estrategias de aprendizaje que dan como resultado un nuevo conocimiento, innovador y de emprendimiento, que a su vez es internalizado por los estudiantes. El objetivo de este artículo fue analizar la

Recibido: 20.03.19 Aceptado: 19.06.19

1 Este artículo forma parte de la producción intelectual de la tesis doctoral titulada: Aplicación de estrategias de aprendizaje basada en la instrucción cognitiva para mejorar el rendimiento académico en la asignatura de matemática en los alumnos de la carrera de computación e informática del instituto de educación superior tecnológico público "nueva esperanza" Trujillo 2016 Perú.

2 Profesor a tiempo completo de la Facultad de Educación y Ciencias de la Comunicación de la Universidad Nacional de Trujillo(UNT)-Perú. Doctor en ciencias de la educación. Licenciado en educación secundaria con especialidad en: Matemática, física y computación. Licenciado en matemáticas. Licenciado en psicología. Correos electrónicos: ramaya@unitru.edu.peamayasauceda@hotmail.com. Miembro investigador de la Red Académica Internacional de Estudios Organizacionales en América Latina, el Caribe e Iberoamérica (REOALCEI)-México. ORCID https://orcid.org/0000-0002-8638-6834

3 Profesora a tiempo completo de la Facultad de Educación y Ciencias de la Comunicación de la Universidad Nacional de Trujillo(UNT)-Perú. Doctora en administración. Doctorado en contabilidad. Estudios en alta investigación posdoctoral en educación, ciencias sociales e interculturalidad. Posdoctorado en comunicación, educación y cultura. Master en política y gestión universitaria. Licenciada en educación, especialidad: Matemática.Miembro investigador de la Red Académica Internacional de Estudios Organizacionales en América Latina, el Caribe e Iberoamérica (REOALCEI)-México. ORCID https://orcid.org/0000-0002-1943-7857 
implementación de estrategias de aprendizaje para mejorar el rendimiento académico de los estudiantes de la asignatura de matemática del instituto de educación superior tecnológico público "Nueva Esperanza", Trujillo - Perú. En esta investigación, se hizo uso de la metodología cuantitativa, lo que permitió describir e interpretar la praxis académica de los alumnos. El estudio realizado consideró el diseño pre experimental con un solo grupo, con una muestra de 48 estudiantes. Se concluye que las estrategias de aprendizaje mejoró el rendimiento académico en la asignatura de matemática.

Palabras clave: Estrategias de aprendizaje, rendimiento académico, aprendizaje, tecnológicos públicos, implementación, educación superior.

\title{
Learning strategies and university academic performance: A look from public technology students
}

\begin{abstract}
Higher education in public technologies, fosters a space of interaction where the ideas that are generated are debated and deepened by teachers, who at the same time make use of learning strategies that result in new, innovative and entrepreneurial knowledge. , which in turn is internalized by students. The objective of this article was to analyze the implementation of learning strategies to improve the academic performance of the students of the mathematics subject of the public technological higher education institute "Nueva Esperanza", Trujillo - Peru. In this research, quantitative methodology was used, which allowed to describe and interpret the academic praxis of the students. The study carried out considered the pre-experimental design with a single group, with a sample of 48 students. It is concluded that learning strategies improved academic performance in the subject of mathematics.
\end{abstract}

Keywords: Learning strategies, academic performance, learning, public technology, implementation, higher education.

\section{Introducción}

Los actuales desafíos sobre formación en educación superior, exigen situar el foco en la evidencia de aprendizajes adquiridos por parte de estudiantes. Lo anterior, repercute en el rol que asumen docentes y estudiantes en el proceso de enseñanza - aprendizaje; Beltrán (1996), entiende que las estrategias de aprendizaje son procedimientos de carácter voluntario 
e intencional, que asume el estudiante para lograr un determinado objetivo. Muchas veces, la implementación de estrategias de autorregulación del aprendizaje dependen, en gran medida, de la capacidad de percepción del estudiante, aunque también de otros factores personales como la motivación por aprender y sobre todo, la percepción que se tiene acerca de la efectividad en el uso de las estrategias de aprendizaje. En ese sentido, Bahamón, Vianchá, Alarcón y Bohórquez (2012) sostienen que, en el ambiente universitario las instituciones con sus docentes deben proporcionarles a sus estudiantes una proximidad a la información del campo complejo, de manera que puedan llegar al procesamiento de la información y del conocimiento, en niveles mejor elaborados.

Aquí, es oportuno señalar que las estrategias de las cuales hacen mayor uso los estudiantes y que redundan en un mejor rendimiento académico, son las metacognitivas (la planificación: control previo del estudio, y la revisión: puesta en marcha del proceso al finalizar). En suma, es comprender el antes (planificación de la meta y los recursos), durante (supervisión y monitorización del proceso) y después (logros alcanzados).

Con los antes expresado, se puede decir, que con la implementación de estrategias de aprendizaje, el estudiante toma conciencia que es el mismo quien tiene el control sobre todo lo que hace, se esfuerza por comprender la exigencia y requisitos de la tarea y actividades a realizar, lo que le permite dar respuesta y soluciones adecuadamente; es capaz de hecho, de planificar y evaluar sus propios trabajos, sus avances y de identificar sus aciertos y dificultades; utilizando las estrategias de estudio más idóneas según sea el caso, valora los logros, reconoce y corrige sus errores(León, Risco y Alarcón, 2014).

En otro orden de ideas, es relevante exponer que según experiencias con los estudiantes, resalta el hecho que para la selección de las estrategias de aprendizaje, el género (masculino o femenino) sí influye para la elección de dichas estrategias, observándose que por ejemplo; en las estrategias de aprendizaje (escalas de: adquisición, codificación, recuperación y de apoyo), son preferidas por los hombres; por otro lado, la preferencia por parte de las mujeres, es hacia la escala de codificación y apoyo. Asimismo, en otros estudios realizados y analizados por Acevedo-Rodríguez (2016), las mujeres mostraron una mayor fortaleza en el uso de estrategias metacognitivas y motivacionales (planificar y revisar sus procesos), en cuanto a los varones su mayor uso fue el de las estrategias cognitivas (memorizar, organizar y elaborar información).

En relación a lo anterior, es oportuno revisar en esta parte, la clasificación de las estrategias de aprendizaje, y motivacionales según Román y Gallego (1994), mediante el instrumento de medición ACRA, establecen cuatro tipos de estrategias: adquisición, codificación, recuperación y apoyo a la información. La primera permite al estudiante analizar como debe aprender la información nueva. La segunda establece la relación entre las ideas principales y secundarias de un texto. La tercera propone los mecanismos para recuperar la información que se almacenó anteriormente y la última, informa sobre los recursos, condiciones que permiten procesar y mejorar el aprendizaje.

Asimismo, en algunas propuestas teóricas se encontró la valoración creciente de modelos centrados en 
el aprendizaje, donde toma fuerza la enseñanza innovadora (trabajo cooperativo, aprendizaje basado en problemas, desarrollo de proyectos, otros), aquí los estudiantes asumen su rol de actores de su propio proceso de aprendizaje, siendo el profesor el mediador o articulador de los entornos y experiencias de dichos aprendizajes (Gargallo, Pérez, Jiménez, Martínez, Giménez, 2017). Según este enfoque, son importantes tanto el método utilizado, en este caso las estrategias de aprendizaje, como el entorno donde se desarrolla el proceso de enseñanza - aprendizaje y en el que se encuentran inmersos los estudiantes.

En el contexto del estudio, uno de los objetivos estratégicos del sistema educativo para los institutos tecnológicos peruanos, es lograr el desarrollo pleno e integral de los estudiantes, sin embargo, la realidad educativa no refleja totalmente esa intencionalidad, porque el proceso educativo, además de ser complejo, presenta limitaciones, entre las cuales se pueden mencionar: la escasa información que poseen sobre teorías cognitivas, así como la inexistencia de propuestas educativas innovadoras que fomenten el aprender a aprender en los educandos.

Teniendo en cuenta que, las estrategias de aprendizaje en el nivel de educación superior están centradas en el estudiante, esto implica un proceso de cimentación del conocimiento mucho más activo y autónomo, dada la madurez del aprendiz; en donde este va regulando sus aprendizajes y seleccionando sus estrategias. Al respecto, Barca-Lozano, Almeida, Porto-Rioboo, Peralbo-Uzquiano y Brenlla-Blanco(2012) sostienen que, el aprendizaje autorregulado se orienta a procesos en los cuales el estudiante dirige metódicamente y controladamente sus operaciones, tendencias y emociones hacia el logro de sus metas planteadas; siendo necesario reconocer el rol que cumplen los estudiantes como agentes activos del sistema educativo universitario.

Por esta razón, las universidades desarrollan currículos con contenidos y objetivos orientados por competencias que fomentan habilidades y actitudes, en relación con el perfil profesional de cada carrera; lo que permite durante el proceso de formación profesional, se tenga en cuenta la evaluación de los estudiantes en el marco de los sistemas de calidad, como una forma de aplicar ciertos estándares, que marquen las pautas para garantizar la adquisición de las competencias necesarias, para desempeñarse con eficiencia y eficacia en el futuro como profesionales. Esto con el propósito de cubrir las necesidades de la demanda social y la demanda del mercado laboral (Blázquez, Chamizo, Cano y Gutiérrez, 2013).

En ese sentido, el educando debe hacerse preguntas para confirmar que en efecto comprende la tarea a realizar, verificando que las estrategias elegidas son las más eficaces, obviamente esto implica que de ser necesario deberá ampliar el tiempo que ha usado para laborar y buscar los recursos suficientes para lograrlo(León, Risco y Alarcón,2014).

En cuanto a la implementación de estrategias de aprendizaje para un mejor rendimiento académico, de los alumnos en educación superior tecnológica, se evidencia muchas veces que,según observaciones sistemáticas de los investigadores de este estudio; está vinculado a la manera de calificar del docente; siendo quien establece parámetros en sus evaluaciones, 
obviando en ocasiones la verdadera función del proceso evaluativo; que implica la aplicación de estrategias de aprendizaje y evaluación adecuadas, lo que trae como consecuencia que los estudiantes tiendan a conformarse con el calificativo mínimo aprobatorio de once (11), por el sólo hecho de estar aprobados en la asignatura; aunado a su nula participación en la selección de las estrategias de evaluación.

Ante estos planteamientos, se formula la siguiente interrogante: ¿En qué medida laimplementación de estrategias de aprendizaje mejora el rendimiento académico de los estudiantes de la asignatura de matemáticas del instituto de educación superior tecnológico público "Nueva Esperanza", Trujillo Perú? Es así como el objetivo de este artículo, fue analizar la implementación de estrategias de aprendizaje para mejorar el rendimiento académico de los estudiantes de la asignatura de matemáticas del instituto de educación superior tecnológico público "Nueva Esperanza", Trujillo - Perú.

Con respecto a otros aspectos del artículo, el procesamiento estadístico, implicó un tratamiento luego de la tabulación de los datos obtenidos y la aplicación de los instrumentos(test) a los sujetos del estudio, con la finalidad de implementar estrategias de aprendizaje en pro del rendimiento académico en los estudiantes del instituto de educación superior objeto de estudio.

Se hizo uso de la estadística descriptiva para la interpretación de los resultados. Asimismo, teniendo en cuenta un nivel de significación de $5 \%$ y haciendo uso del estadístico $z$ para el análisis paramétrico, el cual determinó que existe una diferencia significativa entre los promedios del rendimiento académico en la asignatura de matemática obtenidos al administrar el pre y post test en los estudiantes de la muestra. Se aplicaron los test a una muestra de 48 alumnos; con el propósito de profundizar los hallazgos producto de la observación directa sistemática.

\section{Estrategias de aprendizaje y rendimiento académico universitario: algunas consideraciones teóricas}

Las estrategias de aprendizaje son definidas como secuencias integradas de procedimientos $\mathrm{O}$ actividades mentales activadas con el propósito de facilitar la adquisición, almacenamiento y/o utilización de la información (Nisbett y Shucksmith, 1987), e implican un proceso de toma de decisiones consciente e intencional, sobre qué procedimiento de éstos utilizar para alcanzar determinados objetivos de aprendizaje. Al respecto, Monereo y Pozo (s/f), manifiestan que, las estrategias de aprendizaje de los estudiantes en la escuela deben promover las competencias para el logro de objetivos, con el apoyo del docente, por tanto, es necesario enseñarlas en aras de garantizar un aprendizaje significativo, autónomo, constructivo, colaborativo y eficaz.

En ese sentido, Beltrán(2003) analizado por Gázquez, Pérez, Ruíz, Miras y Vicente (2006:3), lo definen como actividades $u$ operaciones mentales que el estudiante, puede llevar a cabo para facilitar o mejorar la realización de la tarea, haciendo hincapié en dos características esenciales de la estrategia: que sea directa $\mathrm{O}$ indirectamente manipulable y que tenga un carácter propositivo o intencional.

Portanto, siendo que las estrategias de aprendizaje son un conjunto de pautas 
que aplica el estudiante para orientar de modo consciente e intencional sus actividades de aprendizaje, producto de sus experiencias y recomendaciones de compañeros o del profesor, sobre cómo hacer más eficientes sus esfuerzos por aprender; constituyéndose en herramientas fundamentales para la calidad y éxito de las tareas académicas, éstas inducen la actividad reflexiva del estudiante frente al material de estudio en las situaciones de enseñanza y por otra parte son elementos valiosos para el diseño, planificación, ejecución y evaluación de acciones pedagógicas, considerándolos algunos autores como aspectos centrales en el currículum educativo, (Martínez y Galán, 2000).

En el mismo orden de ideas, Avilés y Monjas (2005), sostiene que son acciones específicas tomadas por el estudiante para hacer el aprendizaje más fácil, rápido, disfrutable, auto dirigido, y transferible a nuevas situaciones. Las estrategias de aprendizaje comprenden todo el conjunto de procesos, acciones y actividades que los aprendices pueden desplegar intencionalmente para apoyar y mejorar su aprendizaje ya que son procesos de toma de decisiones conscientes e intencionales en los cuales el alumno elige y recupera, de manera coordinada, los conocimientos que necesita para complementar una determinada demanda $u$ objetivo, dependiendo de las características de la situación educativa.

- Clasificación de las estrategias de aprendizaje en el ámbito académico

Según, Nisbett y Shucksmith (1987), se han identificado cinco tipos de estrategias generalesen el ámbito educativo. Las tres primeras ayudan al alumno a elaborar y organizar los contenidos para que resulte más fácil el aprendizaje (procesar la información), la cuarta está destinada a controlar la actividad mental del alumno para dirigir el aprendizaje y por último, la quinta está de apoyo al aprendizaje para que éste se produzca en las mejores condiciones posibles. A continuación se describen:

a. Estrategias de ensayo: Son aquellas que implicanla repetición activa de los contenidos (diciendo, escribiendo), o centrarse en partes clavesde él. Son ejemplos: Repetir términos en voz alta, reglas nemotécnicas, copiar el material objeto de aprendizaje, tomar notas literales, el subrayado.

b. Estrategias de elaboración: Implican hacer conexiones entre lo nuevo y lo familiar. Por ejemplo: Parafrasear, resumir, crear analogías, tomar notas no literales, responder preguntas (las incluidas en el texto o las que pueda formularse el alumno), describir como se relaciona la información nueva con el conocimiento existente.

\section{c. Estrategias de organización:}

Agrupan la información para que sea más fácil recordarla. Implican imponer estructura a los contenidos de aprendizaje, dividiéndolo en partes e identificando relaciones y jerarquías. Incluyen ejemplos como: Resumir un texto, esquema, subrayado, tabla sinóptico, red semántica, mapa conceptual, árbol ordenado.

\section{d. Estrategias de control de la} comprensión: Estas son las estrategias ligadas a la metacognición. Implican permanecer conscientes de lo que se está tratando de lograr, seguir la pista de las estrategias que se usan y del éxito logrado con ellas y adaptar la conducta en concordancia. Si utilizásemos la metáfora de comparar la mente con un ordenador, estas estrategias actuarían como un procesador central de ordenador. Son un sistema supervisor de la acción y el pensamiento del alumno, 
y se caracterizan por un alto nivel de conciencia y control voluntario.

Entre las estrategias metacognitivas están: la planificación, la regulación y la evaluación,la primera,son aquellas mediante las cuales los alumnos dirigen y controlan su conducta. Son, por tanto, anteriores a que los alumnos realicen ninguna acción. Se llevan a cabo actividades como:

a. Establecer el objetivo y la meta de aprendizaje.

b. Seleccionar los conocimientos previos que son necesarios para llevarla a cabo.

c. Descomponer la tarea en pasos sucesivos.

d. Programar un calendario de ejecución.

e. Prever el tiempo que se necesita para realizar esa tarea, los recursos que se necesitan, el esfuerzo necesario.

f. Seleccionar la estrategia a seguir.

e. Estrategias de regulación, dirección y supervisión: Se utilizan durante la ejecución de la tarea. Indican la capacidad que el alumno tiene para seguir el plan trazado y comprobar su eficacia. Se realizan actividades como: Formular preguntas, seguir el plan trazado, ajustar el tiempo y el esfuerzo requerido por la tarea y por último modificar y buscar estrategias alternativas en el caso de que las seleccionadas anteriormente no sean eficaces.

f. Estrategias de evaluación: Son las encargadas de verificar el proceso de aprendizaje. Se llevan a cabo durante y al final del proceso. Se realizan actividades como: Revisar los pasos dados, valorar si se han conseguido o no los objetivos propuestos, evaluar la calidad de los resultados finales y decidir cuándo concluir el proceso emprendido, cuando hacer pausas, la duración de las pausas, entre otros.

\section{La enseñanza de las estrategias de aprendizaje}

En cuanto a este aspecto, Monereo y Pozo (s/f), plantean la siguiente interrogante: ¿por qué enseñar estrategias de aprendizaje? Como profesor me he preguntado muchas veces, por qué ante una misma clase, unos alumnos aprenden más que otros. ¿Qué es lo que distingue a los alumnos que aprenden bien de los que lo hacen mal?. Existen muchas diferencias individuales entre los alumnos que causan estas variaciones. Una de estas, es la capacidad del alumno para usar las estrategias de aprendizaje: por tanto, enseñar estrategias de aprendizaje a los alumnos, es garantizar el aprendizaje: el aprendizaje eficaz, y fomentar su independencia, (enseñarle a aprender a aprender).El conocimiento de estrategias de aprendizaje por parte del alumno influye directamente en que el alumno sepa, pueda y quiera estudiar.

Es importante destacar, quetambién depende de la disposición de los profesores para hacer de la comprensión en los alumnos del uso correcto de las estrategias, como se aplican, en que momentos, como elegir las más adecuadas, ya que el alumno por sí solo muchas veces no podrá determina estos factores para su aprendizaje y no sabrá a ciencia cierta cómo es el proceso de aprender a aprender; aquí radica como se ha planteado anteriormente el rol mediador de docente.En tanto que los siguientes aspectos:

- Saber: el estudio es un trabajo que debe hacer el alumno, y puede realizarse por métodos que faciliten 
su eficacia. Esto es lo que pretenden las estrategias de aprendizaje: que se llegue alcanzar el máximo rendimiento con menor esfuerzo y más satisfacción personal.

- Poder: para poder estudiar se requiere un mínimo de capacidad o inteligencia. Está demostrado que esta capacidad aumenta cuando se explota adecuadamente y se consigue con las estrategias de aprendizaje.

- Querer: ¿es posible mantener la motivación del alumno por mucho tiempo cuando el esfuerzo (mal empleado por falta de estrategias o por el uso inadecuado de estas) resulta insuficiente?. El uso de buenas y adecuadas estrategias, garantiza que el alumno conozca el esfuerzo que requiere una tarea y que utilice los recursos necesarios específicos e idóneos, para realizarla. Consigue buenos resultados y esto produce que (al conseguir más éxitos) esté más motivado.

De lo descrito anteriormente, sobre los criterios: "saber, poder y querer", es preciso rescatar, que si bien el proceso de aprendizaje es básicamente individual y parte de cualidades o criterios que, en este caso, deben conocer los estudiantes, es necesario tomar en consideración que existen pautas que han de ser manejadas desde el entorno; donde los profesores, a través de la reflexión y las acciones que desempeñan como mediadores, cumplen el rol de enseñar a sus estudiantes a "aprender a aprender"; y a entrenarlos sobre el uso de las estrategias de aprendizaje, pero sobre todo a suministrarles aquellas estrategias que según su naturaleza, puedan aportar mejores y mayores resultados al momento de aprender una materia en específico.

Por lo anterior, un docente con la tarea de mediador, necesariamente debe planificar junto a su grupo de estudiantes, y en consenso con ellos, sobre cuáles y cuantas son las estrategias que funcionarán mejor en el desarrollo de una materia en específico. En cuanto el estudiante se haga dueño y domine las diferentes estrategias, estas pasan a ser las herramientas que le permitirán un mejor desenvolvimiento en su propio proceso de aprendizaje y con resultados más satisfactorios que redundarán en un nivel más aceptable de su rendimiento académico.

Por lo antes expuesto, según Gargallo, Suárez y Ferreras (2007), infieren que un profesor que actúe como mediador es capaz de dirigir diferentes situaciones en el aula donde, en conjunto con los estudiantes, que trabajen para formular y resolver problemas, proponer tareas de diferentes niveles de dificultad, lo cual promueva en éstos la capacidad de indagar, buscar, reflexionar, reconstruir situaciones, que al mismo tiempo sean de ayuda a otros. Siendo muy importante en este proceso, la metodología empleada por el docente, que sea coherente con estos planteamientos y ajustada a los objetivos sobre excelencia que tienen mucha relación con el hecho de cómo enseñar, sin caer en la rutina y muy usada repetición de los saberes y de lo aprendido; tratando en lo posible de integrar diversidad de técnicas y herramientas de fácil asimilación y práctica por parte de los estudiantes.

\section{Rendimiento Académico}

El rendimiento académico, se mide en términos del desempeño demostrado por los alumnos en las áreas del 
conocimiento que se desarrollan en la escuela. Medir el rendimiento académico implica la búsqueda permanente de la calidad educativa, en la medida en que proporciona elementos para el mejoramiento continuo. La teoría sobre el rendimiento académico considera la inteligencia racional como un factor importante en el mismo, pero no es el único; existen otros factores como los ambientales, familiares, sociales, curriculares, extracurriculares y el clima escolar, entre otros.

En consideraciones de Edel (2003:21), el rendimiento escolar es una dimensión fundamental en el proceso de enseñanza-aprendizaje; considerándose como "la capacidad expresada en procesos y resultados para desarrollar tareas escolares, en la que intervienen habilidades y esfuerzo de los estudiantes". En sus investigaciones, analiza los factores socioeconómicos, los créditos de los programas de estudio, los métodos utilizados, los pre saberes de los estudiantes (conocimientos previos); que son los que conforman y constituyen el nivel de pensamiento formal de éstos(Benítez, Jiménez y Osicka, 2000).

Asimismo, González y Tourón (1992), consideran que el rendimiento escolar se puede medir a través de las capacidades de respuesta y las iniciativas manifiestas de lo que un estudiante ha aprendido después de un proceso de formación educativa, por tanto el rendimiento responde a estímulos educativos a partir de la interpretación de objetivos intencionadamente propuestos. Desde una óptica operativa,Herán y Villarroel (1987), plantean que el rendimiento se puede medir por el número de cursos repetidos por un estudiante. De otra manera, Nováez (1986), lo considera como el resultado que se obtiene de una acción académica frente a unos objetivos propuestos.

Por otra parte, el rendimiento académico es definido por Chadwick (1979:34) como "la expresión de capacidades y de características psicológicas del estudiante desarrolladas y actualizadas a través del proceso de enseñanza-aprendizaje que le posibilita obtener un nivel de funcionamiento y logros académicos a lo largo de un período, año o semestre, que se sintetiza en un calificativo final del nivel alcanzado". En el mismo orden de ideas, Escacena y de Frutos (1985) y Andrade, Miranda y Freixas (2000), consideran que el rendimiento académico se puede definir como la aprobación de unos estándares mínimos de conocimientos y aptitudes fijadas por un grupo social determinado.

Es una realidad que el rendimiento académico es considerado un indicador del nivel de aprendizaje que alcanza el estudiante, por lo cual el sistema educativo brinda tanta importancia a dicho indicador. Por lo tanto, se convierte en una medición del aprendizaje logrado en el aula, pasando a ser el objetivo primordial de la educación. En ese sentido, un aspecto que no debe ser olvidado y que debe ser reflexionado, asimilado y llevado a la práctica, en el proceso de enseñanza aprendizaje pasando por la implementación de estrategias de aprendizaje propias, tanto desde el rol de mediador como de parte de los estudiantes, es la presencia en los procesos de la evaluación formativa y continua; lo que va a permitir tanto a estudiantes como profesores, realizar la 
retroalimentación siempre necesaria de los conocimientos o saberes adquiridos y sobre todo de la revisión del proceso, los resultados o productos que se vayan obteniendo; con miras a la excelencia, calidad y a un rendimiento académico óptimo.

\section{Relevancia de las estrategias de aprendizajes y su incidencia en el rendimiento académico: Una análisis desde las}

\section{matemáticas en educación superior}

En esta sección se presentan los resultados arrojados del tratamiento estadístico de los datos y se analizan. Asimismo, se tabulan los datos en cuadros con información de doble entrada con respeto al nivel del rendimiento académico en la aplicación del pre test y post test, considerado en este estudio. A continuación se presentan la siguientes tabla 1 y 2 :

Tabla 1

Distribución de frecuencias de los puntajes obtenidos al aplicar el pre test

\begin{tabular}{ccc}
\hline $\begin{array}{c}\text { Nivel del } \\
\text { rendimiento académico }\end{array}$ & $\begin{array}{c}\text { Frecuencia } \\
\mathrm{n}_{i}\end{array}$ & $\begin{array}{c}\text { Porcentaje } \\
\%\end{array}$ \\
\hline Deficiente & 26 & 54.2 \\
Regular & 16 & 33.3 \\
Bueno & 5 & 10.4 \\
Muy bueno & 1 & 2.1 \\
Total & 48 & 100 \\
\hline
\end{tabular}

Fuente: Elaboración propia a partir de la matriz de datos

Se observa que el $54.2 \%$ de los estudiantes tienen un rendimiento académico deficiente, un $33.3 \%$ de los estudiantes tienen un rendimiento académico regular, un $10.4 \%$ de los estudiantes tienen un rendimiento bueno y un $2.1 \%$ de los estudiantes tienen un rendimiento académico muy bueno. 


\begin{tabular}{lcc}
\multicolumn{4}{c}{ Tabla 2} \\
$\begin{array}{c}\text { Distribución de frecuencias de los puntaj } \\
\text { obtenidos al aplicar el post test }\end{array}$ \\
\hline $\begin{array}{l}\text { Nivel del } \\
\text { rendimiento académico }\end{array}$ & $\begin{array}{c}\text { Frecuencia } \\
\mathrm{n}_{i}\end{array}$ & $\begin{array}{c}\text { Porcentaje } \\
\%\end{array}$ \\
\hline Deficiente & 5 & 10.4 \\
Regular & 14 & 29.2 \\
Bueno & 24 & 50.0 \\
Muy bueno & 5 & 10.4 \\
Total & 48 & 100.0
\end{tabular}

Fuente: Elaboración propia a partir de la matriz de datos

Se observa que el $10.4 \%$ de los estudiantes tienen un rendimiento académico deficiente, un $29.2 \%$ de los estudiantes tienen un rendimiento académico regular, un $50 \%$ de los estudiantes tienen un rendimiento bueno y un $10.4 \%$ de los estudiantes tienen un rendimiento académico muy bueno.

\begin{abstract}
La evaluación antes de implementar las estrategias de aprendizaje, obtuvo un promedio de 8.43 en el rendimiento académico en la asignatura de matemática, considerando un nivel de significación de $5 \%$ y haciendo uso del estadístico z para el análisis paramétrico, tabla 3 .
\end{abstract}

Tabla 3

Promedios de pre test y post test

\begin{tabular}{llll}
\hline Promedios & & Valor Tabular & Valor Experimental \\
\hline Pre test & Post-test & & \\
\hline 8.43 & 16.39 & 16,92 & 5,42 \\
\hline
\end{tabular}

Fuente: Elaboración propia a partir a partir de la matriz de datos

Los resultados obtenidos en el pre test aplicado a la muestra objeto de estudio, para medir el rendimiento académico en la asignatura de Matemática, orientaron para una mayor precisión en la elaboración de una propuesta de estrategias de aprendizaje basadas en la instrucción cognitiva dirigidas a optimizar el rendimiento académico en Matemática. Se tuvo en cuenta que la instrucción cognitiva, en lugar de interpretar a los estudiantes como recipientes vacíos que pueden llenarse con nuevas habilidades, reconoce que los estudiantes vienen a la situación de aprendizaje con 
conocimientos y habilidades previos.

Como se puede observar en la tabla 1 , el $54.2 \%$ del total de alumnos muestran puntajes desaprobatorios y por consiguiente un rendimiento académico deficiente. En tal sentido, la mayoría de los estudiantes no necesariamente muestran haber logrado aprendizajes significativos en la asignatura de Matemática; sin embargo, se trató de aprovechar el bagaje de conocimientos que poseían. Téngase en cuenta que: "El aprendizaje significativo (...) tiene lugar cuando se intenta dar sentido o establecer relaciones entre los nuevos conceptos o nueva información y los conceptos y conocimientos existentes en el alumno o con alguna experiencia anterior"(Ontoria, Molina y de Luque,1996: 16).

La significatividad de los nuevos conocimientos, en cierta forma, está relacionada con el caudal de conocimientos previos que poseen los que aprenden, así como la metodología usada por el docente para motivar y desarrollar un tema. La significatividad potencial del material depende de la significatividad lógica. Según Ontoria, Molina y de Luque (1996: 19), afirma que la "potencial significatividad lógica no sólo depende de la estructura interna del contenido, sino también de la manera como éste sea presentado al alumno".

En cuanto a la relación entre las estrategias de aprendizaje y el rendimiento académico, Pizano (2004), publicó la investigación sobre "Las estrategias de aprendizaje y su relevancia en el rendimiento académico de los alumnos". El mismo, surge en el marco de la investigación "Impacto de las estrategias de aprendizaje en el rendimiento académico de estudiantes del tercer ciclo de la facultad de educación en la Universidad Mayor de San Marcos" (UNMSM)-Perú. La investigación realizada se evaluó con la escala ACRA (escala de estrategias de aprendizaje de José María Ramón Sánchez y Sagrario Gallego Rico). Se trata de cuatro escalas independientes que evalúan el uso que, habitualmente, hacen los estudiantes de siete estrategias de adquisición de información, de cuatro estrategias de recuperación de información y finalmente, de nueve estrategias de apoyo al procesamiento de la información.

Esta investigación concluye que las estrategias de aprendizaje influyen sobre el rendimiento académico, en un $95 \%$ y $99 \%$; en los dos grupos de estudiantes del tercer ciclo de estudios universitarios, siendo relevante estas estrategias como actividad constructiva en la que el sujeto construye su propia representación mental, tomando conciencia de su realidad académica. Además, se estableció una relación estadísticamente significativa entre estrategias de aprendizaje y rendimiento académico, que considera al alumno como agente activo y responsable de la calidad y profundidad de los aprendizajes obtenidos.

El porcentaje de alumnos con un deficiente rendimiento académico en la asignatura de Matemática disminuye significativamente en los resultados del post-test, e inclusive el promedio del posttest se incrementa en aproximadamente 8 puntos, con relación al del pre test. Esto hace pensar en la eficacia del uso de las estrategias de aprendizaje, basadas en la instrucción cognitiva. Lo anterior está relacionado con lo expresado por, Freire (1999), quien estima conveniente indagar si la población estudiantil posee las herramientas necesarias $y$ efectivas para adquirir nuevas formas de aprender, pasando de una mera posición 
de "recipiente de conocimientos" donde por lo general son los docentes quienes "depositan" los saberes o por el contrario asumen el protagonismo de su propio aprendizaje. Esto último sería la acción idónea y por ende lo que produce los resultados anteriores.

Las mediciones efectuadas en el grupo de alumnos de la muestra, a partir de los datos recolectados al aplicar el pre test antes del tratamiento y el post-test después del mismo, han permitido contrastar, afirmándose que existe diferencia significativa entre los promedios en matemática de las mediciones efectuadas antes y después del uso de estrategias de aprendizaje basadas en la instrucción cognitiva, tal como se aprecia la tabla 3 . En la medida que se consideró un nivel de significación de 0,05 y se mantuvieron estables las condiciones medioambientales $y$ otros factores externos e internos, se generaliza los resultados a la población del estudio con una confianza del 95\%.

Teniendo en consideración que las estrategias de aprendizaje están basadas en la instrucción cognitiva, los estudiantes tienen mayor capacidad para procesar la información de manera significativa y a convertirse en seres más independientes. Esto implica que el uso de las estrategias permite ayudarles a construir significados de los materiales informativos, resolver problemas, desarrollar estrategias de pensamiento y asumir la responsabilidad de su aprendizaje significativo, así como transferir habilidades y conceptos a nuevas situaciones.

\section{Conclusiones}

En el instituto de educación superior tecnológico público "Nueva
Esperanza", Trujillo - Perú existe un alto porcentaje de alumnos que desconocen las estrategias de aprendizaje, las cuales pueden hacer uso para su propio proceso de adquisición del conocimiento, todo quedó evidenciado en el pre test, lo que confirma la falta de un tipo de programa o plan dentro de los procesos curriculares que promuevan la adquisición de aprendizajes significativos, relacionados con el área de matemáticas; no se cuenta con la información de cómo aprende el alumno, qué situaciones son más favorables para aprender, cómo procesa la información, cómo responde a estímulos de aprendizaje, para optimizar el proceso de aprender a aprender.

Por consiguiente, se manifiesta un aprendizaje no satisfactorio $y$ un marcado desinterés por aplicar métodos adecuados, además de falta de disciplina en el compromiso de forjar sus propios aprendizajes, lo que trae como consecuencia la nula producción de aprendizajes nuevos y duraderos.

Por último, la aplicación de estrategias de aprendizaje mejora el rendimiento académico en estudiantes de tecnológicos públicos en el Perú; como se confirma en los sujetos de la muestra que presentaron un nivel bueno de su rendimiento académico, luego del tratamiento y uso de estrategias de aprendizaje aplicadas en el post test.

\section{Referencias bibliográficas}

Acevedo-Rodríguez, Maritza (2016), Estrategias de aprendizaje con relación al rendimiento académico y tiempo en alcanzar el grado universitario en enfermería. Facultad de Ciencias de la Salud- Universidad del Málaga, España. Recuperado en :https://riuma.uma.es/xmlui/bitstream/ handle/10630/14346/TD ACEVEDO RODRIGUEZ Maritza.pdf?se- 
quence $=1$ \&isAllowed $=y \quad$ Consultado:02-03- 2019.

Andrade, Miguel; Miranda, Christian y Freixas, Irma (2000), Rendimiento Académico y Variables modificables en alumnos de 2do Medio de Liceos Municipales de la Comuna de Santiago. Santiago de Chile, Chile. http://www.estudiosindigenas.cl/educacion/eaprendizajes rendimiento academico 2 medio santiago.pdf. Consultado:23-06- 2015.

Avilés Martínez, José María y Monjas Casares, Inés (2005). Estudio de incidencia de la intimidación y el maltrato entre iguales en la educación secundaria obligatoria mediante el cuestionario CIMEl (Avilés, 1999)Cuestionario sobre Intimidación y Maltrato Entre Iguales. Anales de Psicología/Annals of Psychology, 21(1), 27-41.Recuperado en:https:// www.um.es/analesps/v21/v21 1/0421 1.pdfConsultado:29-11- 2015.

Bahamón Muñetón, Marly Johana; Vianchá Pinzón, Mildred Alexandra; Alarcón Alarcón, Linda Liliana; Bohórquez Olaya, Claudia Inés(2012),Estilos y estrategias de aprendizaje: una revisión empírica y conceptual de los últimos diez años. Pensamiento Psicológico, vol. 10, núm. 1, pp. 129-144.Pontificia Universidad Javeriana-Cali, Colombia. Recuperado en: http://www.redalyc.org/articulo. oa?id=80124028009 Consultado: 20-02-2016.

Barca-Lozano, Alfonso; Almeida, Leandro S.; Porto-Rioboo, Ana Ma; Peralbo-Uzquiano, Manuel;Brenlla-Blanco, Juan C. (2012), Motivación escolar y rendimiento: impacto de metas académicas, de estrategias de aprendizaje y autoeficacia.Anales de Psicología, vol. 28, núm. 3, octubre, pp. 848-859. Universidad de Murcia-Murcia, España. Recuperado en : http://www.redalyc.org/
articulo.oa?id=16723774023Consultado:23-06- 2018.

Beltrán, Jesús (1996), Procesos, estrategias y técnicas de aprendizaje. Madrid: Síntesis.

Beltrán Llera, Jesús (2003), Estrategias de Aprendizaje. Revista de educación. 332, 55-73. Recuperado en https://dialnet.unirioja.es/servlet/ articulo?codigo $=776715$ Consultado:13-09- 2018.

Benítez, M., Giménez, M., y Osicka, R. (2000), Las asignaturas pendientes y el rendimiento académico: ¿existe alguna relación?. Recuperado en http://www.unne.edu.ar/cyt/humanidades/h-009.pdf Consultado: 05- 092014.

Blázquez Resino, Juan José; Chamizo González, Julián; Cano Montero, Elisa Isabel y Gutiérrez Broncano,Santiago (2013), Calidad de vida universitaria: Identificación de los principales indicadores de satisfacción estudiantil:Revista de Educación, (362), 458-484. Recuperado en: https://recyt.fecyt.es/index.php/Redu/article/ view/14343 Consultado:23-08- 2017.

Chadwick,Clifton (1979), Teorías del aprendizaje para el docente. Tecla.

Edel Navarro, Rubén (2003),El rendimiento académico: concepto, investigación y desarrollo.REICE. Revista Iberoamericana sobre Calidad, Eficacia y Cambio en Educación, vol. 1, núm. 2, julio-diciembre, 2003, p. 0.Red Iberoamericana de Investigación sobre cambio y eficacia escolar; Madrid, España. Recuperado en: http://www.redalyc.org/articulo. oa? id $=55110208$ Consultado en:0504-2019.

Escacena Carrasco, José Luis y de Frutos Reyes, Gregorio (1985), Estratigrafía de la Edad del Bronce en el Monte Berrueco (Medina Sidonia, 
Estrategias de aprendizaje y rendimiento académico universitario: Una mirada desde los estudiantes de tecnológicos públicos

Amaya Sauceda, Rosas Amadeo y Rafael Sánchez, Áurea Elizabeth

Cádiz). Noticiario Arqueológico Hispánico, (24), 7-90.

Freire, Paulo (1999), Pedagogía del Oprimido. México. Siglo Veintiuno Editores.

Gargallo López, Bernardo; Pérez Pérez, Cruz; Jiménez Rodríguez, Miguel Ángel;Martínez Hervás, Noelia; Giménez Beut, Juan Antonio(2017),Métodos centrados en el aprendizaje, implicación del alumno y percepción del contexto de aprendizaje en estudiantesuniversitarios. Revista EducaciónXX1, vol. 20, núm. 2, 2017, pp. 161-187.Universidad Nacional de Educación a Distancia. Madrid, España.Recuperado en: https://www. redalyc.org/pdf/706/70651145007. pdf. Consultado:06-03- 2019.

Gargallo López, Bernardo; Suárez Rodríguez, Jesús y Ferreras Remesal, Alicia(2007),Estrategias de aprendizaje y rendimiento académico en estudiantes universitarios. Revista de Investigación Educativa, vol. 25, núm. 2, 2007, pp. 421-441.Asociación Interuniversitaria de Investigación Pedagógica. Murcia, España. Recuperado en:https://www.redalyc. $\mathrm{org} / \mathrm{pdf} / 2833 / 283321923010 . \mathrm{pdf}-$ Consultado:09-03- 2019.

Gázquez, José J.; Pérez, María del Carmen; Ruiz, María Isabel; Miras, Francisco; Vicente, Florencio (2006), Estrategias de aprendizaje en estudiantes de enseñanza secundaria obligatoria y su relación con laautoestima. International Journal of Psychology and Psychological Therapy, vol. 6, núm. 1, april, 2006, pp. 51-62.Universidad de Almería-Almería, España. Recuperado en: http://www.redalyc.org/articulo. oa? id=56060103 Consultado:09-042019.

González Torres y Tourón Javier (1992), Autoconcepto y rendimiento escolar: sus implicaciones en la motivación y en la autorregulación del aprendizaje. Eunsa.

Herán y Villarroel (1987). Caracterización de algunos factores del alumno y su familia de escuelas urbanas y su incidencia en el rendimiento de castellano y matemáticas en el primer ciclo de enseñanza general básica. Chile: CPEIP, 10.

León Urquijo, Ana Patricia; Risco del Valle, Eduardo; Alarcón Salvo, Cristina(2014),Estrategias de aprendizaje en educación superior en un modelo curricular por competencias. Revista de la Educación Superior, vol. XLIII (4), núm. 172, octubre-diciembre,pp.123-144.Asociación Nacional de Universidades e Instituciones de Educación SuperiorDistrito Federal, México. Recuperado en: http:// www.redalyc.org/articulo.oa?id=60432737006Consultado:29-112015.

Martínez, Reynaldo y Galán, Ferrán (2000), Estrategias de aprendizaje, motivación y rendimiento académico en alumnos universitarios. Revista Española de Orientación y Psicopedagogía, vol. 11, núm. 19,pp. 35-50.Asociación Española de Orientación y Psicopedagogía- España. Recuperado en:http://revistas. uned.es/index.php/reop/article/view/ 11323Consultado:29-11- 2015.

Monereo, Carles y Pozo, Juan Ignacio(s/f), Competencias para (con) vivir con el siglo XXI. Cuadernos de pedagogía, 370(12), 12-18. Recuperado en:http://rubenama. com/articulos/12975732-Monereo-Pozo-Competencias-para-convivir-con-el-siglo-XXI.pdf Consultado:26-09- 2018.

Nisbett y Shucksmith (1987), Estrategias de aprendizaje.

Nováez, M. (1986), Psicología de la 
Actitud del escolar. México: Ed. Iberoamericana.

Ontoria Peña, Antonio; Molina Rubio, Ana, de Luque Sánchez, Angela (1996).Los mapas conceptuales enel Aula. Editorial: Magisterio del Río de la Plata, Argentina.

Pizano Chávez, Guillermina (2004). Las estrategias de aprendizaje y su relevancia en el rendimiento académico de los alumnos. Revista de investi- gación educativa, 8(14), pp.27-30. Universidad Nacional Mayor de San Marcos (UNMSM), Lima-Perú. Recuperado en:https://revistasinvestigacion.unmsm.edu.pe/index. php/educa/article/view/7093/6268n Consultado: 21-10- 2014.

Román Sánchez, José María y Gallego Rico, Sagrario (1994). ACRA. Escalas de estrategias de aprendizaje. Madrid: TEA Ediciones.

- $\quad$ Esta obra está bajo una licencia de Creative Commons Reconocimiento-NoComercial- Compartirlgual 3.0 Unported. http://creativecommons.org/licenses/by-nc-sa/3.0/deed.es_ES 